\title{
Optical activity in diffraction from a planar array of achiral nanoparticles
}

\author{
S. N. Volkov, ${ }^{1, *}$ Ksenia Dolgaleva, ${ }^{2, \dagger}$ Robert W. Boyd, ${ }^{2}$ Konstantins Jefimovs, ${ }^{3,+}$ Jari Turunen, ${ }^{3}$ Yuri Svirko, ${ }^{3}$ \\ Brian K. Canfield, ${ }^{4,}$ and Martti Kauranen ${ }^{4}$ \\ ${ }^{1}$ Department of Physics and Astronomy, University of Rochester, Rochester, New York 14627, USA \\ ${ }^{2}$ The Institute of Optics, University of Rochester, Rochester, New York 14627, USA \\ ${ }^{3}$ Department of Physics and Mathematics, University of Joensuu, 80101 Joensuu, Finland \\ ${ }^{4}$ Department of Physics, Tampere University of Technology, 33101 Tampere, Finland \\ (Received 14 May 2008; revised manuscript received 12 October 2008; published 17 April 2009)
}

\begin{abstract}
We prepare diffractive planar arrays of metal nanoparticles that are chiral because of either the shape of individual particles ("molecular" chirality) or the orientation of achiral particles in the array ("structural" chirality). Both sorts of samples are shown to lead to comparable polarization changes in the diffracted light. For the case of structural chirality, one might assume that these effects can occur only through interparticle interactions, as would be the case for transmission measurements (zero-order diffraction). However, we show that the results can be explained by a simple model in which the polarization effects are based on independent scattering by individual particles, with no interparticle coupling, and with the array structure simply determining the direction of the diffraction maximum. We thus conclude that structural and molecular chiralities are indistinguishable in diffraction experiments.
\end{abstract}

DOI: $10.1103 /$ PhysRevA.79.043819

PACS number(s): 42.25.Fx, 78.67.Bf, 78.20.Ek

\section{INTRODUCTION}

Chiral objects occur in two mirror-image forms (enantiomers) that cannot be superimposed with each other by proper rotations [1]. Chirality is usually associated with molecular structure and leads to optical-activity effects, which arise from different interactions of chiral molecules with leftand right-hand circularly polarized light $[2,3]$. Conventional optical-activity effects, such as circular dichroism and polarization (azimuth) rotation, arise from "molecular" chirality and occur in isotropic bulk liquids (e.g., sugar solutions) and molecular crystals. Because of their molecular origin, these effects are proportional to the density of chiral molecules and build up as light traverses the chiral medium. Optical-activity effects can also arise from a chiral arrangement of achiral objects, e.g., the arrangement of silicon and oxygen atoms in a unit cell of crystalline quartz. Such "structural" chirality vanishes when the crystal is melted or dissolved. Both molecular and structural chiralities thus arise from the threedimensional (3D) nature of the material. It is also possible that materials with neither molecular nor structural chirality give rise to optical-activity effects. This is the case if an experiment is performed where the setup itself is chiral; i.e., it is defined by three noncoplanar vectors with a given handedness. Such effects are known in light scattering from an-

\footnotetext{
*Present address: Department of Electrical and Computer Engineering, The Johns Hopkins University, Baltimore, MD 21218, USA.

†Present address: Department of Electrical and Computer Engineering, University of Toronto, 10 King's College Rd., Toronto, ON M5S 3G4, Canada; ksenia.dolgaleva@utoronto.ca

"Present address: Laboratory for Electronics/Metrology/ Reliability, EMPA, CH-8600 Dübendorf, Switzerland.

${ }^{\S}$ Present address: University of Tennessee Space Institute, 411 B. H. Goethert Pkwy, Tullahoma, TN 37388, USA.
}

isotropic molecules or angular momentum-aligned atoms and in nonlinear optics [4-8]. This possibility, which was also discussed in an early publication regarding parity and optical activity [9], has recently been reintroduced to optics under the label "extrinsically chiral material" [10]. Note also that molecular chirality implies structural chirality, and structural chirality implies chirality of the experimental setup. Therefore, separation of the three different mechanisms may be difficult.

Recent nanofabrication techniques have made it possible to prepare samples with so-called planar or two-dimensional (2D) chirality $[11,12]$. Such samples are usually 2D arrays with a subwavelength period and consist of nanoparticles that cannot be brought into congruence with their mirror image by in-plane rotations or translations. The samples, therefore, possess a sense of twist, which is different when viewed from the front and back sides of the sample. This peculiarity has invoked controversies of possible violation of timereversal symmetry and nonreciprocity of the light-matter interaction in 2D chiral media [13-15]. However, it is now well established that planar arrays of nanoparticles lead to optical activity similar to that of conventional chiral media [16], because of the front-back asymmetry brought about by the substrate [16] or other vertical structure [17], which effectively turns a 2D sample into a 3D sample.

Different diffraction patterns for left- and right-hand circularly polarized light have been observed for planar square gratings consisting of particles with fourfold rotation axis and no reflection symmetry [15]. Following the above classification for bulk media, this result can be seen to arise from 2D molecular chirality because of the particular sense of twist of the individual 2D nanoparticles. Similarly, one can also introduce 2D structural chirality, when the sense of twist of the 2D grating arises from the mutual arrangement of achiral nanoparticles. An attempt has been made to separate contributions from the molecular and structural 2D chiralities to the polarization effects in diffraction experiments [11]. However, no reference sample with pure structural chirality 
was used. Such a sample would have to consist of achiral nanoparticles arranged in a chiral grating with no mirror symmetry. In addition, the experiments were based on nonnormal angle of incidence, which implies low symmetry of the setup, and on varying the polarization of the incident beam, which can distinguish anisotropy from chirality [12], but not different levels of chirality from each other.

In this paper we compare polarization changes in diffraction from planar gratings with pure structural and molecular chiralities. Specifically, we perform polarization measurements for beams diffracted from a grating consisting of achiral crosses, whose mutual orientations make the overall sample 2D chiral, and compare the results with those from a reference sample where the individual particles are chiral. Our experiments are based on a setup with the highest possible symmetry where the incident beam is applied at normal incidence. The incident beam was polarized either parallel or perpendicular to the diffraction plane. We find that the polarization changes from both types of samples are significant and comparable to each other. This is surprising, as the origins of chirality in the two samples are different, and the interparticle coupling would be crucial for observing optical activity in transmission experiments with structurally chiral samples. It is therefore quite natural to expect that the effects may be quantitatively different for samples with chiralities of different origins. We show, however, that in the diffraction experiments this is not the case. Our results therefore suggest that the two types of sample chiralities cannot be separated in diffraction experiments. Our conclusion is further supported by a simple model which describes the polarization of the diffracted wave in terms of light scattering from a strictly $2 \mathrm{D}$ array of nanoparticles. In this model, the grating only enhances scattering into a given diffraction order through constructive interference, as the model involves no interparticle coupling.

\section{EXPERIMENT}

Our sample, fabricated utilizing electron-beam lithography, contains different patterns of nanoparticles on a fused silica substrate. In the direction perpendicular to the sample plane, the particles consist of a $3 \mathrm{~nm} \mathrm{Cr}$ adhesion layer and $30 \mathrm{~nm}$ of $\mathrm{Au}$. The entire structure is coated by $20 \mathrm{~nm}$ protective $\mathrm{SiO}_{2}$ layer. There are eight different nanoparticle patterns (Fig. 1). Patterns 1 and 2 are achiral: pattern 1 is a square lattice of achiral crosses with the legs oriented along the lattice axes, while pattern 2 is a lattice of crosses tilted at $45^{\circ}$ with respect to the lattice axes. Patterns 3 and 4 consist of achiral crosses tilted at $+27.5^{\circ}$ and $-27.5^{\circ}$ with respect to the lattice axes, respectively. The $\pm 27.5^{\circ}$ tilt results in pure structural chirality of the patterns, as the individual particles are achiral. Patterns 5-8 contain chiral gammadions and propellers and, therefore, possess molecular chirality. The chiral patterns in each pair $(3,4),(5,6)$, and $(7,8)$ are enantiomeric forms (mirror images) of each other. The period in all gratings is $800 \mathrm{~nm}$; each pattern is a $1 \times 1 \mathrm{~mm}^{2}$ square.

We illuminated the sample with a linearly polarized light from a $633 \mathrm{~nm}$ He-Ne laser at normal incidence (see Fig. 1). The polarization state of the incident light was controlled by

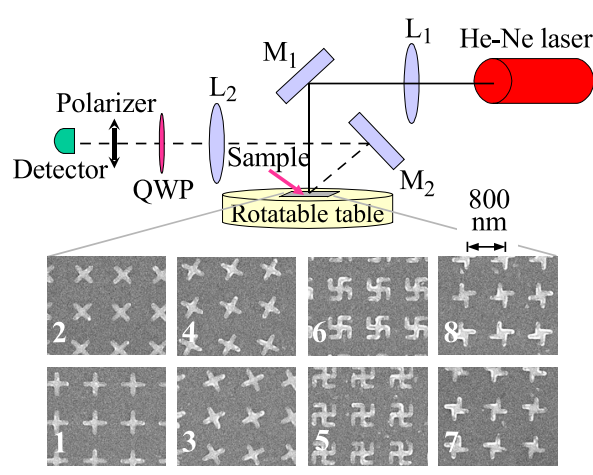

FIG. 1. (Color online) Experimental setup and sample layout.

a half-wave plate and a linear polarizer (not shown in the figure). We measured the polarization rotation and ellipticity of the first-order diffracted beams from all eight patterns for $p$ - and $s$-polarized incident light. (We call the incident light $p$ polarized if its electric field vector lies in the plane perpendicular to the sample and containing the diffracted beam, and $s$ polarized if the electric field is perpendicular to this plane.) Due to the astigmatism of the electron-beam writing, the samples can exhibit some anisotropy that affects the polarization state of the diffracted light. To remove the effects of the residual anisotropy of the structures, we analyzed the polarization states of four equivalent first-order diffracted beams corresponding to the four equivalent azimuthal orientations of the samples, and averaged the results. The spread in the data collected from the four equivalent first-order diffracted beams does not exceed $3^{\circ}$ for patterns 1 and $5-8$, but is about $10^{\circ}$ for patterns $2-4$, as the lattice axes for the latter patterns have a nonzero angle with respect to the directions of the electron-beam writing. We repeated each measurement three times and found that the random errors in our measurements are smaller than the size of the data point in the graphs. However, the precision of the polarizer and the quarter-wave plate rotation is within $1^{\circ}$, which introduces some uncertainty into establishing the "zero level" of ellipticity and polarization rotation. This zero level is set by the achiral patterns 1 and 2 and we have removed this offset from the other results.

The polarization rotation and ellipticity of the diffracted beam are presented in Fig. 2. The achiral patterns 1 and 2 do not change the polarization state of the diffracted light. The chiral patterns 3-8, on the other hand, display clear deviations of the polarization rotation and ellipticity with respect to the zero level established by patterns 1 and 2 . The patterns that are enantiomeric forms of each other demonstrate polarization effects equal in magnitude and opposite in sign, which is a manifestation of chirality. The polarization effects reverse sign as the linear polarization of the incident light changes from $p$ to $s$ state. The strongest polarization changes are observed from the pair of patterns $(5,6)$, which contain chiral gammadions. The effect observed from the pair of patterns $(3,4)$ with pure structural chirality, however, is almost as large. Patterns 7 and 8, consisting of chiral propellers, on the other hand, display very weak polarization changes. 


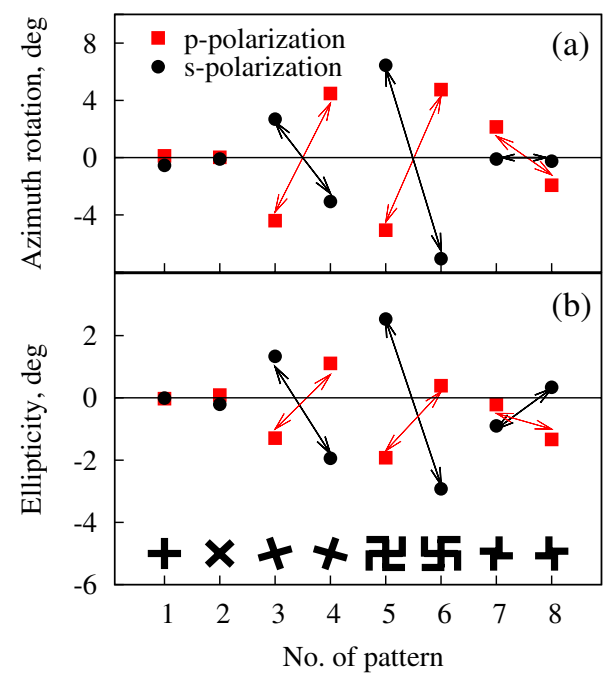

FIG. 2. (Color online) (a) Polarization azimuth rotation and (b) ellipticity of light in diffraction from the planar structures. Data for $p$ - and $s$-polarized incident light are shown as squares and circles, respectively. The results for the two enantiomerically opposite forms are connected by the arrows. The offset of the zero level set by the achiral patterns 1 and 2 is within $1^{\circ}$. It has been shifted to coincide with the zero level of the graphs for both polarization rotation and ellipticity.

\section{WIRE MODEL}

The origin of the polarization effects in patterns 3 and 4 possessing pure structural chirality can be explained by a simple "wire model" (inspired by [11]) where the only possible source of chirality is the orientation of the achiral particles with respect to the square lattice. Let us assume that a plane monochromatic wave with the frequency $\omega$ is incident on a patterned surface with the period $a$ (for convenience, we further do not show the frequency dependence). We consider the linear response of the surface current density $\mathbf{j}(\boldsymbol{\rho})$ to the electric field $\mathbf{E}^{i}(\boldsymbol{\rho})$ of the incident electromagnetic wave, where $\boldsymbol{\rho}$ is a $2 \mathrm{D}$ position vector in the plane of the sample surface:

$$
j_{i}(\boldsymbol{\rho})=\kappa_{i j}(\boldsymbol{\rho}) E_{j}^{i}(\boldsymbol{\rho})
$$

We can treat the linear surface response tensor $\leftrightarrow(\boldsymbol{\rho})$ as a 2 $\times 2$ matrix, as $\mathbf{E}^{i}(\boldsymbol{\rho})$ lies in the surface plane in the case of normal incidence. In order to find the electric field in the far zone that is emitted by the current distribution $\mathbf{j}(\boldsymbol{\rho})$, we need to represent $\mathbf{j}(\boldsymbol{\rho})$ as a spatial Fourier series. Since $\mathbf{E}^{i}(\boldsymbol{\rho})$ is homogeneous in the surface plane, the problem reduces to a spatial Fourier representation of $\overleftrightarrow{\kappa}(\boldsymbol{\rho})$. As the sample is periodic in both main directions of the square lattice (which we call $X$ and $Y$ directions), we can write it down as

$$
\kappa_{i j}(\boldsymbol{\rho})=\sum_{\alpha \beta} \widetilde{\kappa}_{i j}^{(\alpha, \beta)} \exp \left[i \frac{2 \pi}{a}\left(\alpha \rho_{x}+\beta \rho_{y}\right)\right],
$$

where

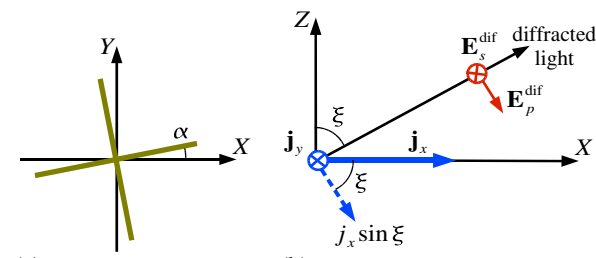

(a)

(b)

FIG. 3. (Color online) Scattering from a tilted cross. (a) Top view of the cross. (b) Side view of the scattering geometry.

$$
\tilde{\boldsymbol{\kappa}}_{i j}^{(\alpha, \beta)}=\frac{1}{S} \int_{S} \kappa_{i j}(\boldsymbol{\rho}) \exp \left[-i \frac{2 \pi}{a}\left(\alpha \rho_{x}+\beta \rho_{y}\right)\right] d \boldsymbol{\rho} .
$$

The zeroth order of expansion (3), $\widetilde{\kappa}_{i j}^{(0,0)} \equiv \bar{\kappa}_{i j}$, describes the reflected wave. The term with $\tilde{\kappa}_{i j}^{(1,0)}$ describes the first order of diffraction in the direction of positive $x$, i.e., one of the four first-order diffraction maxima in the reflection geometry.

We model each "molecule" with a cross made of two wires of length $2 d$ each. The cross is rotated counterclockwise by an angle $\alpha$ with respect to the $X Y$ coordinate system of the square lattice [see Fig. 3(a)]. As evident from standard diffraction theory, the waves emitted by different unit cells are coherently added only in the directions of the diffraction maxima determined by the lattice period $a$. We consider the scattering of light from the pattern in the direction of the first diffraction maximum $(1,0)$, which lies in the $X Z$ plane and has the angle of diffraction $\xi$ [see Fig. 3(b)]. The electric field $\mathbf{E}^{\text {dif }}$ in the far zone in that direction is determined by the respective spatial Fourier harmonic of $\mathbf{j}(\boldsymbol{\rho})$, which can be written as

$$
\widetilde{j}_{m}^{(1,0)}=\widetilde{\kappa}_{m n}^{(1,0)} E_{n}^{i} .
$$

A straightforward calculation yields the result

$$
\overleftrightarrow{\widetilde{\kappa}^{(1,0)}}=\sigma \frac{a}{\pi}\left[\begin{array}{ll}
\beta \cos \alpha+\gamma \sin \alpha & \beta \sin \alpha-\gamma \cos \alpha \\
\beta \sin \alpha-\gamma \cos \alpha & \beta \frac{\sin ^{2} \alpha}{\cos \alpha}+\gamma \frac{\cos ^{2} \alpha}{\sin \alpha}
\end{array}\right] .
$$

Here $\sigma$ is the specific linear conductivity of the wires, $\beta$ $=\sin [(\pi d / a) \cos \alpha]$, and $\gamma=\sin [(\pi d / a) \sin \alpha]$. The $s$ component of diffracted light, $\mathbf{E}_{s}^{\mathrm{dif}}$, is collinear with the $Y$ axis, while its $p$ component, $\mathbf{E}_{p}^{\mathrm{dif}}$, makes the angle $\xi$ with the $X$ axis (see Fig. 3). Therefore, we can find the components $\mathbf{E}_{s}^{\text {dif }}$ and $\mathbf{E}_{p}^{\mathrm{dif}}$ from $\mathbf{E}_{s}^{\mathrm{dif}}=C \widetilde{j}_{y}^{(1,0)}$ and $\mathbf{E}_{p}^{\mathrm{dif}}=C \widetilde{j}_{x}^{(1,0)} \sin \xi$, where $C$ is a constant that is identical for both $\mathbf{E}_{s}^{\text {dif }}$ and $\mathbf{E}_{p}^{\text {dif }}$. These results then directly yield the polarization rotation.

In Fig. 4 we show the theoretical polarization rotation of the diffracted electric field as a function of the tilt angle $\alpha$ of the wire cross with respect to the lattice axes for the $p$ - and $s$-polarized incident light. The graphs are plotted for the leg length of the crosses $2 d=500 \mathrm{~nm}$ and the lattice period $a$ $=800 \mathrm{~nm}$, which approximately corresponds to the geometry of our samples. The case of $\alpha= \pm 27.5^{\circ}$ corresponds to our patterns 3 and 4 (shown as data points in the same graph). It is clear that the predictions of the wire model are in good agreement with the respective experimental data. In particu- 


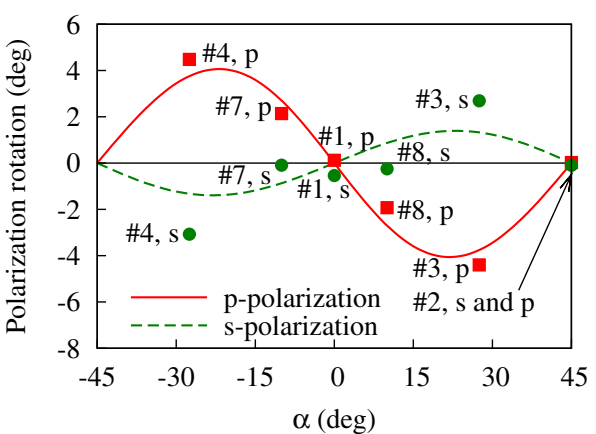

FIG. 4. (Color online) Polarization azimuth rotation of the diffracted light as a function of the tilt angle $\alpha$ of the crosses with respect to the lattice axes, calculated using the wire model. The solid and dashed curves correspond to the $p$ and $s$ polarizations of the incident light, respectively.

lar, the wire model predicts a smaller effect for the $s$-polarized incident light compared to that for the $p$-polarized incident light, which we observed experimentally. Patterns 7 and 8, which are chiral propellers, show a very weak polarization rotation and elliptization. These patterns resemble achiral crosses tilted with respect to the lattice axes by a very small angle. From Fig. 1 we can estimate the tilting angle to be about $10^{\circ}$, with the tilting directions of patterns 7 and 8 being the same as those of patterns 4 and 3, respectively. We also plotted the data points corresponding to patterns 7 and 8 in Fig. 4. The predictions of our wire model for this tilting angle agree well with our experimental data.

The wire model illustrates that the 2D structural chirality manifests itself in the optical activity of diffracted light, similar to that from pure 2D molecular chirality. This occurs even for highly symmetric normal incidence. For achiral particles, the sample is chiral whenever a distinct symmetry direction of each particle is tilted with respect to the lattice axes of the sample. For chiral particles, the sample is always chiral, because the individual particles possess no symmetry direction, which to compare with the lattice axes. Consequently, attempts to separate structural from molecular chirality in a sample consisting of chiral particles would be meaningless. This is in agreement with the observation that polarization effects are qualitatively determined by the overall symmetry of the sample, not the details of how this symmetry arises [9].

The similar polarization effects for both molecular and structural chiralities can also be understood as manifestations of the chirality of the diffraction setup. Such chirality arises from the shape and orientation of a single nanoparticle in the setup, with the lattice only defining the directions of nonzero diffraction orders. The origin of the chirality of the setup can be also understood from a crystallographic prospective. Indeed, the 2D point symmetry group of both kinds of our chiral samples is $p 4$, which lacks the in-plane mirrorreflection operation. Although the normal-incidence geometry in our experiment is as symmetric as it can be, the diffraction direction is off-normal as determined by the symmetry of the lattice. These factors give rise to the leftright asymmetry required for observing the polarization effects. It is important to keep in mind, however, that these symmetry-based considerations cannot give quantitative predictions, as they are not based on a detailed microscopic analysis and, in particular, do not include any assumptions about interparticle coupling. Thus, our experimental results and their analysis help to build a more detailed understanding of the polarization effects.

\section{CONCLUSIONS}

In conclusion, we have reported the observation of polarization rotation and elliptization of light diffracted from planar arrays consisting of either chiral or achiral nanoparticles. We have shown that in both cases, the polarization changes are comparable. They are present even for non-mirrorsymmetric patterns consisting of achiral particles (i.e., possessing pure structural chirality). Our experimental data are in good agreement with the predictions of a simple wire model that describes light scattering from a planar achiral particle and includes no interparticle coupling. Our analysis of the data and the model suggests that diffraction experiments cannot distinguish between the polarization effects arising due to molecular and structural chiralities of the samples. Our findings also demonstrate that in practical applications, patterns with pure structural chirality can be as efficient as those consisting of chiral particles, while having less pattern complexity and thus being easier to fabricate. Artificial planar structures with pure structural chirality can thus be considered as a whole new promising class of materials to be used in polarization control devices. This conclusion is not limited to metal-based structures considered in the present paper, but is also true for all-dielectric planar chiral structures [18], which may be promising because of lower losses and which will be a subject of a separate study.

\section{ACKNOWLEDGMENTS}

We acknowledge discussions with T. Verbiest and R. Serimaa. This work was supported by the Academy of Finland Foundation (Grant Nos. 111701, 209806, 113245, 114913, and 115781), and by the Nanophotonics Research and Development Program funded by the Ministry of Education of Finland.
[1] S. Bassiri, C. H. Papas, and N. Engheta, J. Opt. Soc. Am. A 5, 1450 (1988).

[2] J. B. Biot, Mem. Acad. Sci. Inst. Fr. 13, 39 (1835).

[3] J. B. Biot, Bull. Soc. Philomath. 190 (1815).
[4] J. R. Appling, M. G. White, T. M. Orlando, and S. L. Anderson, J. Chem. Phys. 85, 6803 (1986).

[5] C. Westphal, J. Bansmann, M. Getzlaff, and G. Schönhense, Phys. Rev. Lett. 63, 151 (1989). 
[6] N. Chandra, Phys. Rev. A 39, 2256 (1989).

[7] R. L. Dubs, S. N. Dixit, and V. McKoy, Phys. Rev. Lett. 54, 1249 (1985).

[8] T. Verbiest, M. Kauranen, Y. Van Rompaey, and A. Persoons, Phys. Rev. Lett. 77, 1456 (1996).

[9] L. D. Barron, Nature (London) 238, 17 (1972).

[10] E. Plum, V. A. Fedotov, and N. I. Zheludev, Appl. Phys. Lett. 93, 191911 (2008).

[11] A. Papakostas, A. Potts, D. M. Bagnall, S. L. Prosvirnin, H. J. Coles, and N. I. Zheludev, Phys. Rev. Lett. 90, 107404 (2003).

[12] T. Vallius, K. Jefimovs, J. Turunen, P. Vahimaa, and Y. Svirko, Appl. Phys. Lett. 83, 234 (2003).

[13] A. S. Schwanecke, A. Krasavin, D. M. Bagnall, A. Potts, A. V. Zayats, and N. I. Zheludev, Phys. Rev. Lett. 91, 247404
(2003).

[14] W. Zhang, A. Potts, and D. M. Bagnall, J. Opt. A, Pure Appl. Opt. 8, 878 (2006).

[15] S. L. Prosvirnin and N. I. Zheludev, Phys. Rev. E 71, 037603 (2005).

[16] M. Kuwata-Gonokami, N. Saito, Y. Ino, M. Kauranen, K. Jefimovs, T. Vallius, J. Turunen, and Y. Svirko, Phys. Rev. Lett. 95, 227401 (2005).

[17] M. Decker, M. W. Klein, M. Wegener, and S. Linden, Opt. Lett. 32, 856 (2007).

[18] K. Konishi, B. Bai, X. Meng, P. Karvinen, J. Turunen, Y. P. Svirko, and M. Kuwata-Gonokami, Opt. Express 16, 7189 (2008). 